\title{
Microchip Transfer Process for Implantable Flexible Bioelectronic Devices
}

Julien Martens, Laboratory for Biomedical Microtechnology of Microsystems Technology (IMTEK) at the Albert-Ludwigs-University, Freiburg i. Brsg., Germany, julien.martens@imtek.uni-freiburg.de Calogero Gueli (calogero.gueli@,imtek.uni-freiburg.de), Dr. Max Eickenscheidt (max.eickenscheidt@,imtek.uni-freiburg.de), Prof. Dr.-Ing. Thomas Stieglitz (stieglitz@imtek.de), Laboratory for Biomedical Microtechnology of Microsystems Technology (IMTEK) at the Albert-Ludwigs-University, Freiburg i. Brsg., Germany

\section{Introduction}

The demands on flexible implants for recording and stimulating neural signals have increased in recent years with regard to their functionality and spatial resolution. These requirements can be met by embedding powerful complementary metal oxide semiconductor (CMOS) microchips into thin biocompatible polymer substrates. So-called chip-in-foil systems thus combine mechanical properties of a polymer substrate and performance of CMOS technology.

\section{Methods}

The development of a process for direct transfer of multiple CMOS microchips (edge length $<400 \mu$ m) simultaneously into thin polyimide (PI) substrates is subject of this study. It allows the use of standard microelectromechanical systems (MEMS) processes for further levelled superficial layer build-up. This is achieved with the help of a carrier silicon wafer equipped with cavities for precise chip placement and a sacrificial layer to facilitate release of the chip-in-foil systems. In a post-processing step all silicon chips are thinned down to $100 \mu \mathrm{m}$.

\section{Results}

With this process a transfer yield of $100 \%(n=34)$ could be achieved. Chip rotational error on substrates could be determined to be as low as $0.21^{\circ} \pm 0.10^{\circ}$. Die adhesion was examined by shear tests, resulting in shear strength of 58.1 $\mathrm{MPa} \pm 13.7 \mathrm{MPa}$, which dropped to $15.2 \mathrm{MPa} \pm 10.5 \mathrm{MPa}$ after accelerated ageing in $60{ }^{\circ} \mathrm{C}$ phosphate buffered saline solution (PBS) for 16 days.

\section{Conclusion}

This study demonstrates a reliable microchip transfer process with low positioning error into flexible PI substrates with post-processing thinning of the dies. The use of a carrier silicon wafer allows precise electrical interconnect fabrication with standard MEMS processing techniques and without handling of thin and fragile chips. These results are a prerequisite to meet needs of reliability and structural biocompatibility in implantable flexible bioelectronics devices. 


\title{
Concept for stretchable implantable neural interfaces entirely made of PDMS
}

\author{
Yara Baslan, Thomas Stieglitz*, Patrick Kiele* \\ Laboratory for Biomedical Microtechnology, IMTEK, University of Freiburg, Freiburg, 79110 Germany \\ E-mail (yara.baslan@imtek.uni-freiburg.de)
}

*T.S. and P.K. are joint last authors.

\section{Introduction}

Recent advances in material development and process technologies provide new possibilities for improving the production of PDMS-based neural interfaces. The state-of-the-art interfaces consist of a combination of PDMS substrates and metal foil as electrically conductive material for tracks. The combination of materials with mismatched Young's moduli limits the mechanical properties, the longevity and integrity of the implants. This work presents a concept for the use of PDMS with conductive filler material to replace the metal foil and produce a mechanically homogeneous structure. Further, a fabrication process for this structure is developed.

\section{Methods}

The fabrication technique varied from the conventional methods in that it used a printing system for all stages, instead of the need for expensive machinery, such as a laser cutting system. Further, time-consuming handwork was reduced to a minimum. For this process, materials were selected based on their mechanical properties, printability, and curing properties. The printing followed a layered, top-down approach, meaning that the first printed layer on the substrate became the top layer after releasing the finished electrode. The approach involved the printing of uncured conductive PDMS on metal contact disc sites to achieve adhesion as curing progressed. The conducting structures were embedded in a printed insulating matrix.

\section{Results}

We identified process parameters to successfully produce fully printed neural interfaces with electrical conducting silicone embedded in insulating PDMS. Due to printing approach, the process is fully capable for rapid prototyping. Further, the required machinery was reduced to a printing setup only. In addition, the processing time and required handwork were highly reduced with the printing process.

\section{Conclusion}

Printing has shown to be an alternative to photolithography and laser structuring for medium feature sizes and integration levels of neural electrode arrays. PDMS as an established material of medical devices met the requirement of both, insulator and conductor part of the device, depending on the detailed material properties. Flexibility and stretchability of the samples increased in comparison to standard neural interfaces using metal as conductor lines. This approach thereby paves the way for more robust and long-term stable neural interfaces. 


\title{
Next Generation of Implantable Pressure Sensor to Measure Liquor Pressure of a Ventricular Drainage System with sub hPa accuracy
}

\author{
Michael Görtz, Fraunhofer-Institut für Mikroelektronische Schaltungen und Systeme (IMS), Duisburg, Germany, \\ Michael.Goertz@,ims.fraunhofer.de \\ Christian Walk, Fraunhofer-Institut für Mikroelektronische Schaltungen und Systeme (IMS), Duisburg, Germany, \\ Christian.Walk@ims.fraunhofer.de \\ Norbert Haas, Fraunhofer-Institut für Mikroelektronische Schaltungen und Systeme (IMS), Duisburg, Germany, \\ Norbert.Haas@ims.fraunhofer.de \\ Christian Gleumes, Christoph Miethke GmbH \& Co. KG, Potsdam, Germany, Christian.Gleumes@miethke.com \\ Andreas Bunge, Christoph Miethke GmbH \& Co. KG, Potsdam, Germany, Andreas.Bunge@miethke.com \\ Karsten Seidl, Fraunhofer Institute for Microelectronic Circuits and Systems, Duisburg, Germany and Department of \\ Electronic Components and Circuits and Center for Nanointegration Duisburg-Essen (CENIDE), University of Duisburg- \\ Essen, Duisburg, Germany, karsten.seidl@ims.fraunhofer.de
}

\section{Introduction}

The human brain is filled with cerebrospinal fluid (CSF), which is produced in the ventricles. Normally, production and reabsorption are balanced. Patients suffering from normal pressure hydrocephalus produce more CSF during resorption. This leads to an increase in intracranial pressure.

This article describes the development of an new application-specific integrated circuit suitable for monolithic integration of a Micro-Electro-Mechanical pressure sensor array on the ASIC. This system can be used to measure pressure in a implantable ventricular drainage system.

\section{Methods}

Towards the development of a fully integrated IC with micromachined capacitive pressure sensor elements on CMOS, a high-precision readout (RO)-IC is developed in $180 \mathrm{~nm}$ CMOS node. The ROIC has a specific planarization suitable for post-processing. An 18-bit analog-to-digital converter is developed as second-order low pass sigma-delta modulator in a feedback structure implemented in switched capacitor circuit technology.

The ROIC has a total area of less than $4.5 \mathrm{~mm}^{2}$.

\section{Results}

As a preliminary result, the ROIC achieves more than $105 \mathrm{dBc}$ spurious-free dynamic range in a $200 \mathrm{~Hz}$ band-width while consuming only $1.35 \mathrm{~mW}$. Micromachined capacitive pressure sensors were wire bonded to the ROIC and characterized. The sensor system was calibrated in pressure range from $650 \mathrm{hPa}$ to $1250 \mathrm{hPa}$ and in a temperature range from $18^{\circ} \mathrm{C}$ to $42^{\circ} \mathrm{C}$. The $1 \sigma$ noise is less than $0.05 \mathrm{hPa}$ and the achieved accuracy is better than $0.2 \mathrm{hPa}(<0.16 \mathrm{mmHg})$. An integrated temperature sensor allows for $0.05 \mathrm{~K}$ accuracy.

\section{Conclusion}

In corporation with the Christoph Miethke GmbH \& Co KG the ROIC was developed for wireless measurements of the cerebrospinal fluid pressure in the shunt. The developed pressure sensor ROIC allows previously unattained accuracies for pressure and temperature measurements in medical implants and is suitable for integration into passive transponder systems.

This publication is partly funded by the Federal Ministry of Education and Research under the project reference numbers 16FMD01K, 16FMD02 and 16FMD03. The results were partly obtained in a project funded by the German Federal Ministry of Education and Research under grant number 13GW0275A and 13GW0275B. 


\section{Pancreatic Implant for Glucose Monitoring}

René von Metzen, NMI Naturwissenschaftliches und Medizinisches Institut, Markwiesenstr. 55, 72770 Reutlingen

Udo Kraushaar, NMI Naturwissenschaftliches und Medizinisches Institut, Markwiesenstr. 55, 72770 Reutlingen

Cor Scherjon and Zili Yu, Institut für Mikroelektronik Stuttgart, Allmandring 30a, 70569 Stuttgart

Martin Schuettler and Maria Sousa, Cortec GmbH, Neuer Messplatz 3, 79108 Freiburg

Andreas Kirschniak, Kliniken Maria Hilf GmbH, Viersener Straße 450, 41063 Mönchengladbach

Christian Kempe and Bernd Lecher, mfd Diagnostics GmbH, Mikroforum Ring 5, 55234 Wendelsheim

Andreas Pfützner, PFÜTZNER Science \& Health Institute GmbH, Haifa-Allee 20, 55128 Mainz

\section{Introduction}

According to statistics from WHO, more than 400 million people worldwide suffer from diabetes mellitus, and the number is rising. Most of them suffer from type 2 diabetes, caused by a combination of reduced insulin sensitivity and production in the body. Beta cells within the islets of Langerhans in the pancreas secrete insulin. There is a direct correlation between the electrical activity of beta cells and the extracellular glucose concentration.

\section{Methods}

In the BMBF-funded project 'PanaMEA' (grant number 13GW0397), a pancreatic implant is being developed that measures this electrical activity with a microelectrode array (MEA). The measured signals are processed in an ASIC and transmitted wirelessly to an extracorporeal module. Signal analysis is carried out in situ, so that only small amounts of data need to be transmitted.

The interface between the delivery system and the pancreas is realised by a flexible MEA. The design aims to slide electrodes under the organ skin place it there. Via a flexible lead area, a mechanical decoupling is realised to the other end of the MEA, which is connected to the hermetic housing. During the development phase, the MEA will be tested for functionality in vitro using islands of Langerhans.

In the project, a hermetically sealed miniature housing is being developed to protect the implanted electronics from body fluid, into which the highly integrated electronic circuits (signal amplification, digitisation and wireless communication and power supply) are sealed. The particular challenge is the combination of the small housing size, the requirement for tightness and the high number of feedthroughs that connect the electrical contacts of the MEA with the sealed-in electronics.

\section{Conclusion}

The system's output shall be used as control for an insulin pump. This approach should enable long-term and continuous blood glucose measurement and thus revolutionise the therapy of diabetes. 


\section{Adhesion Testing between Various Silicon Surfaces and an Ultra-Con- formable Medical Grade Silicone for Hybrid Implant Packaging}

Benedikt Szabo, Laboratory for Biomedical Microtechnology of Microsystem Technology (IMTEK) at the Albert-Ludwigs-University, Freiburg i. Brsg., Germany, benedikt.szabo@imtek.uni-freiburg.de

Radu Diaconescu (radu.diaconescu@mars.uni-freiburg.de), Thomas Stieglitz (stieglitz@imtek.de), Laboratory for Biomedical Microtechnology of Microsystem Technology (IMTEK) and BrainLinks-BrainTools Center at the Albert-Ludwigs-University, Freiburg i. Brsg., Germany

\section{Introduction}

Flexible encapsulation is an integral part for microimplants and a challenge for every fabrication process. Adhesion between the encapsulation and the implant substrate is of paramount importance, especially when the encapsulation is covering a moving surface, e.g. an implanted ultrasound transducer, hence strong bonds are required between the encapsulant and the surface. Furthermore, the encapsulation must be applied as gently as possible so that the encapsulated surfaces are neither deformed nor damaged.

\section{Materials and Methods}

Analysis of the adhesion between various silicon-based surfaces and medical grade silicone are subject of this study. The surfaces investigated are low temperature PECVD fabricated silicon dioxides $\left(\mathrm{SiO}_{2}\right)$, silicon nitride ( $\left.\mathrm{SiN}\right)$ and silicon carbide (SiC); the investigated silicone is MED-1000, a RTV silicone adhesive. Previously, to achieve the greatest possible bond, these specimens were placed in partially cured silicone and weighted down. In our approach the specimens were placed on freshly spin-coated silicone without any additional weight, the substrate was Alumina Dioxide $\left(\mathrm{Al}_{2} \mathrm{O}_{3}\right)$. To determine the adhesive forces, a shear test was performed by shearing off the silicon surfaces.

\section{Results}

The tested samples showed a good bonding strength of up to $8 \mathrm{MPa}$ between the different silicon surfaces and the silicone adhesive. It was further observed that the tested samples exhibited cohesion failure from a size of $20 \mathrm{~mm}^{2}$ before they were sheared off.

\section{Conclusion}

In summary, adhesion bonds, most likely chemical bonds, are formed between the silicon surfaces and the silicone. These adhesive forces are sufficient for many flexible implants and encapsulate the implants by intrinsic connections between implant and encapsulation. 


\section{Plasma Enhanced Atomic Layer Deposition of Iridium Oxide for Ap- plication in Miniaturized Neural Implants}

Nicolai Simon, Institute for Microsystems Technology (iMST), Faculty of Mechanical \& Medical Engineering, Furtwangen University, Furtwangen, Germany, n.simon@hs-furtwangen.de

Maria Asplund, BrainLinks-BrainTools Center \& Department of Microsystems Engineering (IMTEK), Albert-LudwigUniversity of Freiburg), Freiburg, Germany, maria.asplund@,imtek.uni-freiburg.de

Thomas Stieglitz, BrainLinks-BrainTools Center \& Laboratory for Biomedical Microsystems, Department of Microsystems Eng.-IMTEK, Albert-Ludwig-University of Freiburg, Freiburg, Germany, stieglitz@imtek.uni-freiburg.de

Volker Bucher, Institute for Microsystems Technology (iMST), Faculty of Mechanical \& Medical Engineering, Furtwangen University, Furtwangen, Germany,

Volker.Bucher@hs-furtwangen.de

\section{Introduction}

High quality recording of neuronal activities and electrical stimulation require neurotechnical implants with appropriate electrode material. Iridium oxide $\left(\mathrm{IrO}_{\mathrm{X}}\right)$ is an excellent choice of material due to its biocompatibility, low electrochemical impedance, superior charge injection capacity, corrosion resistance, longevity, and electrochemical stability. This material can be deposited by sputtering, for example. However, the target material to deposit such films is highly expensive and the conformity is strongly limited. Structures below 10 microns are difficult to coat homogeneously. Plasma enhanced atomic layer deposition (PE-ALD) and a suitable precursor could be a promising technique to produce highly conformal and performant $\mathrm{IrO}_{\mathrm{X}}$-films at low temperatures and low costs. Various studies have reported the deposition of iridium oxide, but usually at very high temperatures. These processes are not suitable for polymer substrates and limits the use of such post processing together with active implants.

\section{Methods}

In this work the (Methylcyclopentadienyl)(1,5-cyclooctadiene) iridium(I) (MeCp (Ir) COD) precursor was used as a promising approach for depositing $\mathrm{IrO}_{\mathrm{X}}$-films using low temperature PE-ALD. This precursor is normally used for chemical vapour deposition processes. First experiments were carried out on silicon substrates at deposition temperatures of $110 \mathrm{C}^{\circ}$. The precursor was heated up to $75^{\circ} \mathrm{C}$ and oxygen plasma was used as co-reactant. The deposited films were analysed with EDX and AFM.

\section{Results}

Film growth could be achieved with this precursor (MeCp (Ir) COD). AFM images showed cauliflower-like growth on the silicon substrates and EDX analysis confirmed that the films were $\mathrm{IrO}_{\mathrm{X}}$.

\section{Conclusion}

First experiments showed that (MeCp (Ir) COD) could be a promising precursor for producing $\mathrm{IrO}_{\mathrm{X}}$ films on structures smaller than 10 microns. Further experiments are carried out to define the ALD window and coated microelectrodes will be tested for their electrochemical stability and performance as a material for recording and stimulation. 